\title{
General Abbreviations
}

\begin{tabular}{|c|c|c|c|}
\hline abstr. & abstract & loc. & locative \\
\hline acc. & accusative & Luw. & Luwian \\
\hline act. & active & Lyc. & Lycian \\
\hline adj. & adjective & masc. & masculine \\
\hline adv. & adverb & med. & middle \\
\hline Aeol. & Aeolic & MS / MSS & manuscript, manuscripts \\
\hline Akk. & Akkadian & Myc. & Mycenaean \\
\hline aor. & aorist & nom. & nominative \\
\hline Att.-Ion. & Attic-Ionic & ntr. & neuter \\
\hline $\mathrm{AV}$ & Atharvaveda & OCS & Old Church Slavonic \\
\hline Avest. & Avestan & $\mathrm{OH}$ & Old Hittite \\
\hline Balt. & Baltic & Ol & Old Irish \\
\hline Boeot. & Boeotian & Olnd. & Old Indian \\
\hline $\mathrm{Br}$. & Brahmana & perf. & perfect \\
\hline Bret. & Breton & pers. & person \\
\hline Bulg. & Bulgarian & PIE & Proto-Indo-European \\
\hline caus. & causative & pl. & plural \\
\hline cent. & century & plupf. & pluperfect \\
\hline Class. & Classical & PN & personal name \\
\hline cod. & codex & Pol. & Polish \\
\hline Comm. Slav. & Common Slavic & pres. & present \\
\hline dat. & dative & ptcpl. & participle \\
\hline denom. & denominative & Russ. & Russian \\
\hline Dor. & Doric & RV & Rggveda \\
\hline Eng. & English & schol. & scholium / scholia \\
\hline fut. & future & sing. & singular \\
\hline gen. & genitive & Skt. & Sanskrit \\
\hline Ger. & German & s.l. & supra lineam \\
\hline Gk. & Greek & subj. & subjunctive \\
\hline Goth. & Gothic & subst. & substantive \\
\hline Hitt. & Hittite & suff. & suffix \\
\hline Ice. & Icelandic & Sum. & Sumerian \\
\hline IE & Indo-European & SV & Sāmaveda \\
\hline imperat. & imperative & s.v. & sub voce \\
\hline impf. & imperfect & Tarent. & Tarentine \\
\hline Ind. & Indian & Thess. & Thessalian \\
\hline intr. & intransitive & TS & Taittirīya Saṃhita \\
\hline Ir. & Irish & Umbr. & Umbrian \\
\hline It. & Italian & Ved. & Vedic \\
\hline Lat. & Latin & v.l. / vv.ll. & varia lectio / variae lec- \\
\hline Latv. & Latvian & & tiones \\
\hline
\end{tabular}


